\title{
Integrodifferential Equations on Time Scales with Henstock-Kurzweil-Pettis Delta Integrals
}

\author{
Aneta Sikorska-Nowak \\ Faculty of Mathematics and Computer Science, Adam Mickiewicz University, Umultowska 87, \\ 61-614 Poznań, Poland \\ Correspondence should be addressed to Aneta Sikorska-Nowak, anetas@amu.edu.pl \\ Received 11 February 2010; Accepted 17 June 2010 \\ Academic Editor: H. Bevan Thompson \\ Copyright (C) 2010 Aneta Sikorska-Nowak. This is an open access article distributed under the \\ Creative Commons Attribution License, which permits unrestricted use, distribution, and \\ reproduction in any medium, provided the original work is properly cited. \\ We prove existence theorems for integro-differential equations $x^{\Delta}(t)=f\left(t, x(t), \int_{0}^{t} k(t, s, x(s)) \Delta s\right)$, \\ $x(0)=x_{0}, t \in I_{a}=[0, a] \cap T, a \in R_{+}$, where $T$ denotes a time scale (nonempty closed subset of \\ real numbers $R$ ), and $I_{a}$ is a time scale interval. The functions $f, k$ are weakly-weakly sequentially \\ continuous with values in a Banach space $E$, and the integral is taken in the sense of Henstock- \\ Kurzweil-Pettis delta integral. This integral generalizes the Henstock-Kurzweil delta integral \\ and the Pettis integral. Additionally, the functions $f$ and $k$ satisfy some boundary conditions \\ and conditions expressed in terms of measures of weak noncompactness. Moreover, we prove \\ Ambrosetti's lemma.
}

\section{Introduction}

A time scale $T$ is a nonempty closed subset of real numbers $R$, with the subspace topology inherited from the standard topology of $R$.

The three most popular examples of calculus on time scales are differential calculus, difference calculus, and quantum calculus (see Kac and Cheung [1]), that is, when $T=R$, $T=N, T=q^{N_{0}}=\left\{q^{t}: t \in N_{0}\right\}$, where $q>1$.

Time scale (or a measure chain) was introduced by Hilger in his Ph.D. thesis in 1988, [2]. Since the time Hilger formed the definitions of a derivative and integral on a time scale, several authors have extended on various aspects of the theory [3-11]. Time scales have been shown to be applicable to any field that can be described by means of discrete or continuous models.

In this paper we consider an integrodifferential equation. As is known, ordinary integrodifferential equations, an extreme case of integrodifferential equations on time scales [12-21], find many applications in various mathematical problems; see Corduneanu's book [22] and references therein for details. 
In [23] the authors extend such results to the integrodifferential equations on time scales and therefore obtained corresponding criteria which can be employed to study the difference equation of Volterra type [21, 24], $q$-difference equations of Volterra type, and so forth.

In [25] the authors proved a new comparison result and develop the monotone iterative technique to show the existence of extremal solutions of the periodic boundary value problems of nonlinear integrodifferential equation on time scales.

We extend this result by proving the existence of a pseudosolution of the problem

$$
\begin{aligned}
x^{\Delta}(t) & =f\left(t, x(t), \int_{0}^{t} k(t, s, x(s)) \Delta s\right), \quad t \in I_{a}=[0, a] \cap T, a \in R_{+}, \\
x(0) & =x_{0}
\end{aligned}
$$

where $f: I_{a} \times E \times E \rightarrow E, k: I_{a} \times I_{a} \times E \rightarrow E, x: I_{a} \rightarrow E, T$ denotes a time scale, $0 \in T, I_{a}$ denotes a time scale interval, $(E,\|\cdot\|)$ is a Banach space, and $x^{\Delta}$ denotes the pseudo $\Delta$ derivative of $x$.

We use a new (Henstock-Kurzweil-Pettis- $\Delta$-integral), more general concept of integral on time scales. This new integral generalizes the Henstock-Kurzweil delta integral, which has been studied by Peterson and Thompson in [26], Avsec et al. in [27] and the Pettis integral [28]. These integrals are important when we consider the weak topology on a Banach space.

The Henstock-Kurzweil delta integral contains the Riemann delta, Lebesgue delta and Bochner delta integrals as special cases. These integrals will enable time scale researchers to study more general dynamic equations. In [26] it is shown that there are highly oscillatory functions that are not delta integrable on a time scale, but are Henstock-Kurzweil delta integral.

Let us remark that the existence of the Henstock-Kurzweil integral over $[a, b]$ implies the existence of such integrals over all subintervals of $[a, b]$ but not for all measurable subsets of this interval, so the theory of such integrals on $T$ does not follows from general theory on $R$.

In [29] Cichoń introduce a definition of the Henstock-Kurzweil delta integral (HK- $\Delta$ integral) and HL delta integral (HL- $\Delta$-integral) on Banach spaces for checking the existence of solutions of differential (or dynamic) equations in Banach spaces. He presented also a new definition of the Henstock-Kurzweil-Pettis delta integral on time scales.

The study for weak solutions of Cauchy differential equations in Banach spaces was initiated by Szép [30] and theorems on the existence of weak solutions of this problem were proved by Cramer et al. [31], Kubiaczyk [32], Kubiaczyk and Szufla [33], Mitchell and Smith [34], Szufla [35], and Cichoń and Kubiaczyk [36]. There are also some existence theorems for the Volterra and Urysohn integral equations [37] on time scales.

Similar methods for solving existence problems for difference equations in Banach spaces equipped with its weak topology were studied, for instance, in [38-40]. In particular, the importance of conditions expressed in terms of the weak topology was considered in [38].

We will unify both cases and using the weak topology, we will obtain the first result for pseudosolutions of an integrodifferential dynamic problem. (This is new also for $q$-difference equations.)

Our result extends the existence of pseudosolutions not only to the discrete intervals with uniform step size $(h Z)$ but also to the discrete intervals with nonuniform step size $\left(K_{q}\right)$. 
We note that (1.1) in its general form involves some different types of differential and difference equations depending on the choice of the time scale $T$. For example:

(1) for $T=R$, we have $\sigma(t)=t, \mu(t)=0$, and $x^{\Delta}(t)=x^{\prime}(t)$, and (1.1) becomes the Cauchy integrodifferential equation:

$$
\begin{gathered}
x^{\prime}(t)=f\left(t, x(t), \int_{0}^{t} k(t, s, x(s)) d s\right), \quad t \in R, \\
x(0)=x_{0}
\end{gathered}
$$

(2) for $T=N$, we have $\sigma(n)=n+1, \mu(n)=1$, and $x^{\Delta}(n)=\Delta x(n)=x(n+1)-x(n)$, and (1.1) becomes the Cauchy problem:

$$
\begin{gathered}
\Delta x(t)=f\left(t, x(t), \int_{0}^{t} k(t, s, x(s)) d s\right), \quad t \in N, \\
x(0)=x_{0} .
\end{gathered}
$$

We assume that the functions $f, k$ are weakly-weakly sequentially continuous with values in a Banach space and satisfy some regularity conditions expressed in terms of the De Blasi measure of weak noncompactness. We introduce a weakly sequentially continuous operator associated to an integral equation which is equivalent to (1.1). There exist many important examples of mappings which are weakly sequentially continuous but not weakly continuous. The relations between weakly sequentially continuous and weakly continuous mappings are studied by Ball [41].

Adopting the fixed point theorem for weakly sequentially continuous mappings given by Kubiaczyk [42] and the properties of measures of weak noncompactness, we are able to study the existence results for problem (1.1)

\section{Preliminaries}

Let $(E,\|\cdot\|)$ be a Banach space and let $E^{*}$ be the dual space. Moreover, let $\left(C\left(I_{a}, E\right), \omega\right)$ denote the space of all continuous functions from $I_{a}$ to $E$ endowed with the topology $\sigma\left(C\left(I_{a}, E\right), C\left(I_{a}, E\right)^{*}\right)$ and $C_{\mathrm{rd}}\left(I_{a}, E\right)$ denotes the space of all rd-continuous functions from the time scale interval $I_{a}$ to $E$.

By $\mu_{\Delta}$ we denote the Lebesgue measure on $T$. For a precise definition and basic properties of this measure we refer the reader to [43].

We now gather some well-known definitions and results from the literature, which we will use throughtout this paper.

Definition 2.1 (see [44]). A family $\mathbb{F}$ of functions $F$ is said to be uniformly absolutely continuous in the restricted sense on $A \subseteq[a, b]$ or in short uniformly $A C_{*}(A)$ if for every $\varepsilon>0$ there exists $\eta>0$ such that for every $F$ in $\mathbb{F}$ and for every finite or infinite sequence of nonoverlapping intervals $\left\{\left[a_{i}, b_{i}\right]\right\}$ with $a_{i}, b_{i} \in A$ and satisfying $\sum_{i} \mu_{\Delta}\left(\left[a_{i}, b_{i}\right]\right)<\eta$, one has $\sum_{i} \omega\left(F,\left[a_{i}, b_{i}\right]\right)<\varepsilon$ where $\omega$ denotes the oscillation of $F$ over $\left[a_{i}, b_{i}\right]$ (i.e., $\omega\left(F,\left[a_{i}, b_{i}\right]\right)=$ $\left.\sup \left\{|F(r)-F(s)|: r, s \in\left[a_{i}, b_{i}\right]\right\}\right)$. 
A family $\mathbb{F}$ of functions $F$ is said to be uniformly generalized absolutely continuous in the restricted sense on $[a, b]$ or uniformly $\mathrm{ACG}_{*}$ if $[a, b]$ is the union of a sequence of closed sets $A_{i}$ such that on each $A_{i}$ the function $F$ is uniformly $A C_{*}\left(A_{i}\right)$.

Definition 2.2. A function $f: I_{a} \rightarrow E$ is said to be weakly continuous if it is continuous from $I_{a}$ to $E$ endowed with its weak topology. A function $g: E \rightarrow E_{1}$, where $E$ and $E_{1}$ are Banach spaces, is said to be weakly-weakly sequentially continuous if for each weakly convergent sequence $\left(x_{n}\right) \subset E$, the sequence $\left(g\left(x_{n}\right)\right)$ is weakly convergent in $E_{1}$. If a sequence $x_{n}$ tends weakly to $x_{0}$ in $E$ we will denote it by $x_{n} \stackrel{\omega}{\rightarrow} x_{0}$.

Theorem 2.3 (see [42]). Let E be a metrizable locally convex topological vector space. Let $D$ be a closed convex subset of $E$, and let $F$ be a weakly-weakly sequentially continuous map of $D$ into itself. If for some $x \in D$ the implication

$$
\bar{V}=\overline{\operatorname{conv}}(\{x\} \cup F(V)) \Longrightarrow V \text { is relatively weakly compact, }
$$

holds for every subset $V$ of $D$, then $F$ has a fixed point.

(I) To understand the so-called dynamic equations and follow this paper easily, we present some preliminary definitions and notations of time scales which are very common in the literature (see [3-5, 7-11] and references therein).

A time scale $T$ is a nonempty closed subset of real numbers $R$, with the subspace topology inherited from the standard topology of $R$. If $a, b$ are points in $T$, then a time scale interval $I$ we denote by $I=[a, b]=\{t \in T: a \leq t \leq b\}$ and $I_{a}=\{t \in T: 0 \leq t \leq a\}$. Other types of intervals are approached similarly. By a subinterval $I_{b}$ of $I_{a}$ we mean the time scale subinterval.

Definition 2.4. The forward jump operator $\sigma: T \rightarrow T$ and the backward jump operator $\rho:$ $T \rightarrow T$ are defined by $\sigma(t)=\inf \{s \in T: s>t\}$ and $\rho(t)=\sup \{s \in T: s<t\}$, respectively. We put $\inf \emptyset=\sup T$ (i.e., $\sigma(M)=M$ if $T$ has a maximum $M$ ) and $\sup \emptyset=\inf T$ (i.e., $\rho(m)=m$ if $T$ has a minimum $m$ ). The jump operators $\sigma$ and $\rho$ allow the classification of points in time scale in the following way: $t$ is called right dense, right scattered, left dense, left scattered, dense and isolated if $\sigma(t)=t, \sigma(t)>t, \rho(t)=t, \rho(t)<t, \rho(t)=t=\sigma(t)$ and $\rho(t)<t<\sigma(t)$, respectively.

Definition 2.5. One says that $k: T \rightarrow E$ is right-dense continuous (rd-continuous) if $k$ is continuous at every right-dense point $t \in T$ and $\lim _{s \rightarrow t^{-}} k(s)$ exists and is finite at every leftdense point $t \in T$.

Definition 2.6. Fix $t \in T$. Let $f: I \rightarrow E$. Then one defines the $\Delta$-derivative $f^{\Delta}(t)$ by

$$
f^{\Delta}(t)=\lim _{s \rightarrow t} \frac{f(\sigma(t))-f(s)}{\sigma(t)-s} .
$$

Remark 2.7. The $\Delta$-derivative satisfies

(1) $f^{\Delta}=f^{\prime}$ is the usual derivative if $T=R$,

(2) $f^{\Delta}=\Delta f$ is the usual forward difference operator if $T=Z$,

(3) $f^{\Delta}=D_{q} f$ is the $q$-derivative if $T=q^{N_{0}}=\left\{q^{t}: t \in N_{0}\right\}, q>1$. 
Hence, time scales allows us unify the treatment of differential and difference equations (and many more time scales).

(II) We need to define some integrals which are important, when we consider the weak topology on a Banach space $E$.

We will use the notation $\eta(t):=\sigma(t)-t$, where $\eta$ is called the graininess function and $\vartheta(t):=t-\rho(t)$, where $\vartheta$ is called the left-graininess function.

We say that $\delta=\left(\delta_{L}, \delta_{R}\right)$ is a $\Delta$-gauge for time scale interval $[a, b]$ provided $\delta_{L}(t)>0$ on $(a, b], \delta_{R}(t)>0$ on $[a, b), \delta_{L} \geq 0, \delta_{R} \geq 0$ and $\delta_{R} \geq \eta(t)$ for all $t \in[a, b)$.

We say that a partition $D$ for a time scale interval $[a, b]$ given by

$$
D=\left\{a=t_{0} \leq \xi_{1} \leq t_{1} \leq \cdots \leq t_{n-1} \leq \xi_{n} \leq t_{n}=b\right\}
$$

with $t_{i}>t_{i-1}$ for $1 \leq i \leq n$ and $t_{i}, \xi_{i} \in T$ is $\delta$-fine if

$$
\xi_{i}-\delta_{L}\left(\xi_{i}\right) \leq t_{i-1}<t_{i} \leq \xi_{i}+\delta_{R}\left(\xi_{i}\right) \text { for } 1 \leq i \leq n
$$

Definition 2.8 (see [29]). A function $f:[a, b] \rightarrow E$ is Henstock-Kurzweil- $\Delta$-integrable on $[a, b](\Delta$-HK integrable in short) if there exists a function $F:[a, b] \rightarrow E$, defined on the subintervals of $[a, b]$, satisfying the following property: given $\varepsilon>0$ there exists a positive function $\delta$ on $[a, b]$ such that $D=\{[u, v], \xi\}$ is $\delta$-fine division of a $[a, b]$, one has

$$
\left\|\sum_{D} f(\xi)(v-u)-(F(v)-F(u))\right\|<\varepsilon .
$$

Definition 2.9 (see [29]). A function $f:[a, b] \rightarrow E$ is Henstock-Lebesgue- $\Delta$-integrable on $[a, b](\Delta$-HL integrable in short) if there exists a function $F:[a, b] \rightarrow E$, defined on the subintervals of $[a, b]$, satisfying the following property: given $\varepsilon>0$ there exists a positive function $\delta$ on $[a, b]$ such that $D=\{[u, v], \xi\}$ is $\delta$-fine division of a $[a, b]$, one has

$$
\sum_{D}\|f(\xi)(v-u)-(F(v)-F(u))\|<\varepsilon
$$

Remark 2.10. We note that, by the triangle inequality, if $f$ is $\Delta$-HL integrable it is also $\Delta$-HK integrable. In general, the converse is not true. For real-valued functions, the two integrals are equivalent.

Definition 2.11 (see $[28,29]$ ). The function $f: I_{a} \rightarrow E$ is Pettis- $\Delta$-integrable $((P)$ integrable for short) if

(1) $\forall x^{*} \in E^{*}, x^{*} f$ is Lebesgue- $\Delta$-integrable on $I_{a}$,

(2) $\forall A \subset I, \exists g \in E, \forall x^{*} \in E^{*}, x^{*} g=(\Delta-L) \int_{A} x^{*} f(s) \Delta s$.

Now, we present a new definition of the integral on time scales which is a generalization for both the Pettis- $\Delta$-integral and the Henstock-Kurzweil- $\Delta$-integral. 
Definition 2.12 (see [29]). The function $f: I_{a} \rightarrow E$ is Henstock-Kurzweil-Pettis- $\Delta$-integrable (HKP- $\Delta$-integrable for short) if

(1) $\forall x^{*} \in E^{*}, x^{*} f$ is Henstock-Kurzweil- $\Delta$-integrable on $I_{a}$,

(2) $\forall t \in I_{a} \forall x^{*} \in E^{*}, x^{*} g(t)=(\Delta-\mathrm{HK}) \int_{0}^{t} x^{*} f(s) \Delta s$.

The function $g$ will be called a primitive of $f$ and by $g(t)=(\Delta-\mathrm{HKP}) \int_{0}^{t} f(s) \Delta s$ we will denote the Henstock-Kurzweil-Pettis- $\Delta$-integral of $f$ on the interval $I_{a}$.

In [29] the authors give examples of Henstock-Kurzweil-Pettis- $\Delta$-integrable functions which are not integrable in the sense of Pettis and Henstock-Kurzweil on time scales.

Theorem 2.13 (see [29] mean value theorem). For each $\Delta$-subinterval $[c, d] \subset[a, b]$, if the integral $(\Delta-H K P) \int_{c}^{d} y(s) \Delta s$ exists, then one has

$$
(\Delta-H K P) \int_{c}^{d} y(s) \Delta s \in \mu_{\Delta}([c, d]) \cdot \overline{\operatorname{conv}} y([c, d]),
$$

where $\overline{\operatorname{conv}} y([c, d])$ denotes the close convex hull of the set $y([c, d])$.

Theorem 2.14 (see [45]). Let $f_{n}, f: I_{a} \rightarrow$ E and assume that $f_{n}: I_{a} \rightarrow$ E are $\Delta$-HKP integrable on $I_{a}$. Let $F_{n}$ be a primitive of $f_{n}$. If one assumes that:

(1) $\forall x^{*} \in E^{*}, x^{*} f_{n}(t) \rightarrow x^{*} f(t) \mu_{\Delta}$ a.e. on $I_{a}$

(2) for each $x^{*} \in E^{*}$ the family $G=\left\{x^{*} F_{n}: n=1,2, \ldots\right\}$ is uniformly $A C G_{*}$ on $I_{a}$ (i.e., weakly uniformly $A C G_{*}$ on $I_{a}$ ),

(3) for each $x^{*} \in E^{*}$ the set $G$ is equicontinuous on $I_{a}$,

then $f$ is $\Delta$-HKP integrable on $I_{a}$ and $\int_{0}^{t} f_{n}(s) \Delta s$ tends weakly in $E$ to $\int_{0}^{t} f(s) \Delta s$ for each $t \in I_{a}$.

Theorem 2.15 (see [5] Gronwall's inequality). Suppose that $u, g, h \in C_{\mathrm{rd}}\left(I_{a}, E\right)$ and $h \geq 0$. Then

$$
u(t) \leq g(t)+\int_{0}^{t} h(\tau) u(\tau) \Delta \tau, \text { for each } t \in I_{a}
$$

implies that

$$
u(t) \leq\left(g(t)+\int_{0}^{t} g(\tau) h(\tau) \Delta \tau\right) \exp \left(\int_{0}^{t} h(\tau) \Delta \tau\right), \text { for each } t \in I_{a} .
$$

(III) The De Blasi measure of weak noncompactness is one of our fundamental tool in this paper (see [46]).

Let $A$ be a bounded nonempty subset of $E$.

The measure of weak noncompactness $\beta(A)$ is defined by

$$
\beta(A)=\inf \left\{t>0: \text { there exists } C \in K^{\omega} \text { such that } A \subset C+t B_{0}\right\} \text {, }
$$

where $K^{\omega}$ is a set of weakly compact subsets of $E$ and $B_{0}$ is a norm unit ball in $E$. 
Some properties of the measure of weak noncompactness $\beta(A)$ are known [46]:

(1) if $A \subset B$, then $\beta(A) \leq \beta(B)$;

(2) $\beta(A)=\beta\left(\overline{A^{w}}\right)$, where $\overline{A^{w}}$ denotes the weak closure of $A$;

(3) $\beta(A)=0$ if and only if $A$ is relatively weakly compact;

(4) $\beta(A \cup B)=\max \{\beta(A), \beta(B)\}$;

(5) $\beta(\lambda A)=|\lambda| \beta(A),(\lambda \in R)$;

(6) $\beta(A+B) \leq \beta(A)+\beta(B)$;

(7) $\beta(\overline{\operatorname{conv}}(A))=\beta(\operatorname{conv}(A))=\beta(A)$, where $\operatorname{conv}(A)$ denotes the convex hull of $A$.

We will need the following lemmas.

Lemma 2.16 (see [47]). Let $E_{1}, E_{2}$ be bounded subsets of the Banach space $E$. If $\left\|E_{1}\right\|=\sup \{\|x\|$ : $\left.x \in E_{1}\right\}<1$, then

$$
\beta\left(E_{1}+E_{2}\right) \leq \beta\left(E_{2}\right)+\left\|E_{1}\right\| \beta\left(K\left(E_{2}, 1\right)\right),
$$

where $K\left(E_{2}, 1\right)=\left\{x: D\left(E_{2}, x\right)<1\right\}$ and $D\left(E_{2}, x\right)=\inf \left\{\|x-y\|: y \in E_{2}\right\}$.

The lemma below is an adaptation of the corresponding result of Ambrosetti (see [48]).

Lemma 2.17. Let $H \subset C\left(I_{a}, E\right)$ be a family of strongly equicontinuous functions. Let $H(t)=\{h(t) \in$ $E, h \in H\}$, for $t \in I_{a}$ and $H\left(I_{a}\right)=\bigcup_{t \in I_{a}} H(t)$. Then

$$
\beta_{C}(H)=\sup _{t \in I_{a}} \beta(H(t))=\beta\left(H\left(I_{a}\right)\right)
$$

where $\beta_{C}(H)$ denotes the measure of weak noncompactness in $C\left(I_{a}, E\right)$ and the function $t \mapsto \beta(H(t))$ is continuous.

Proof. (I) First we prove the equality: $\sup _{t \in I_{a}} \beta(H(t))=\beta\left(H\left(I_{a}\right)\right)$.

Since $H(t) \subset H\left(I_{a}\right)$ by the first property of the measure of noncompactness $\beta(H(t)) \leq$ $\beta\left(H\left(I_{a}\right)\right)$ and consequently

$$
\sup _{t \in I_{a}} \beta(H(t)) \leq \beta\left(H\left(I_{a}\right)\right)
$$

By strong equicontinuity of $H$ we deduce that for $\varepsilon>0$, there exists $\delta>0$ such that $|t-s|<$ $\delta \Rightarrow\|u(t)-u(s)\|<\varepsilon$ for $t, s \in I_{a}$ and for all $u \in H$. 
We divide the interval $I_{a}=\{t \in T: 0 \leq t \leq a\}$ in the following way: $t_{0}=0$,

$$
\begin{gathered}
t_{1}=\sup _{s \in I_{a}}\left\{s: s \geq t_{0}, s-t_{0}<\delta\right\}, \\
t_{2}=\sup _{s \in I_{a}}\left\{s: s \geq t_{1}, s-t_{1}<\delta\right\}, \\
\vdots \\
t_{n}=\sup _{s \in I_{a}}\left\{s: s \geq t_{n-1}, s-t_{n-1}<\delta\right\} .
\end{gathered}
$$

Since $T$ is closed, we have $t_{i} \in I_{a}$. If some $t_{i+1}=t_{i}$ then $t_{i+2}=\inf \left\{t \in T: t \geq t_{i+1}\right\}$.

As

$$
u(t)=u\left(t_{i}\right)+u(t)-u\left(t_{i}\right) \in u\left(t_{i}\right)+\varepsilon K(0,1)
$$

where $K(0,1)=\{x:\|x\|<1\}$, we have

$$
u(t) \in \bigcup_{i=1}^{n} H\left(t_{i}\right)+\varepsilon K(0,1), \quad H\left(I_{a}\right) \subset \bigcup_{i=1}^{n} H\left(t_{i}\right)+\varepsilon K(0,1) .
$$

By the properties of the measure of weak noncompactness and Lemma 2.16, we obtain

$$
\begin{aligned}
\beta\left(H\left(I_{a}\right)\right) & \leq \beta\left(\bigcup_{i=1}^{n} H\left(t_{i}\right)\right)+\|\varepsilon K(0,1)\| \cdot \beta\left(K\left(\bigcup_{i=1}^{n} H\left(t_{i}\right), 1\right)\right) \\
& <\sup _{t_{i} \in I_{a}} \beta\left(H\left(t_{i}\right)\right)+\varepsilon \beta\left(K\left(H\left(I_{a}\right), 1\right)\right) \\
& \leq \sup _{t \in I_{a}} \beta(H(t))+\varepsilon \beta\left(K\left(H\left(I_{a}\right), 1\right)\right) .
\end{aligned}
$$

Since the above inequality holds, for any $\varepsilon>0$, we have

$$
\beta\left(H\left(I_{a}\right)\right) \leq \sup _{t \in I_{a}} \beta(H(t)) .
$$

Hence, from (2.13) and (2.18), we conclude that $\beta\left(H\left(I_{a}\right)\right)=\sup _{t \in I_{a}} \beta(H(t))$.

(II) The proof of the equality $\beta_{C}(H)=\sup _{t \in I_{a}} \beta(H(t))$ is similar to the proof of Lemma 2.1 of Ambrosetti (see [48]), where we choose points $t_{i}$ as in part (I) of our proof.

(III) Now we prove that the function $t \mapsto \beta(H(t))$ is continuous.

Let $v(t)=\beta(H(t))$ because $H(t) \subset H(t) \dot{-} H(s)+H(s) \subset H(t) \dot{-} H(s)+H(s)$, where $H(t) \dot{-} H(s)+H(s)=\{y(t): y(t)=y(t)-y(s)+y(s): y \in H\}$ and the sum is taken in the Minkowski sense.

By the property (vi) of the measure of weak noncompactness, we have

$$
\beta(H(t)) \leq \beta(H(t) \dot{-} H(s))+\beta(H(s)) .
$$


This implies that

$$
\begin{aligned}
|\beta(H(t))-\beta(H(s))| & \leq \beta(H(t) \dot{-} H(s)) \leq \delta(H(t) \dot{-} H(s)) \\
& =\sup _{x, y \in H}\{\|(x(t)-x(s))-(y(t)-y(s))\|\} \\
& \leq \sup _{x, y \in H}\{\|x(t)-x(s)\|+\|y(t)-y(s)\|\} .
\end{aligned}
$$

By equicontinuity of $H$ we obtain the continuity of $v(t)$.

\section{Main Results}

Now, we will consider equivalently integral problem

$$
x(t)=x_{0}+\int_{0}^{t} f\left(z, x(z), \int_{0}^{z} k(z, s, x(s)) \Delta s\right) \Delta z, \quad t \in I_{a}=[0, a] \cap T
$$

where $f: I_{a} \times E \times E \rightarrow E, k: I_{a} \times I_{a} \times E \rightarrow E, x: I_{a} \rightarrow E, T$ denotes a time scale, $0 \in T, I_{a}$ denotes a time scale interval, $(E,\|\cdot\|)$ is a Banach space, and integrals are taken in the sense of $\Delta$-HKP integrals.

Fix $x^{*} \in E^{*}$ and consider the problem

$$
\left(x^{*} x\right)^{\Delta}=x^{*}\left(f\left(t, x(t), \int_{0}^{t} k(t, s, x(s))\right) \Delta s\right) .
$$

Let us introduce a definition.

Definition 3.1. Let $F: I \rightarrow E$ and let $A \subset I$. The function $f: A \rightarrow E$ is a pseudo $\Delta$-derivative of $F$ on $A$ if for each $x^{*} \in E^{*}$ the real-valued function $x^{*} F$ is $\Delta$-differentiable $\mu_{\Delta}$ almost everywhere on $A$ and $\left(x^{*} F\right)^{\Delta}=x^{*} f \quad \mu_{\Delta}$ almost everywhere on $A$.

Regarding the above definition it is clear that the left-hand side of (3.2) can be rewritten to the form $x^{*}\left(x^{\Delta}\right)$, where $x^{\Delta}$ denotes the pseudo $\Delta$-derivative. solution.

To obtain the existence result for our problem it is necessary to define a notion of a

Definition 3.2. A function $x: I_{a} \rightarrow E$ is said to be a pseudosolution of the problem (1.1) if it satisfies the following conditions:

(1) $x(\cdot)$ is $\mathrm{ACG}_{*}$ function,

(2) $x(0)=x_{0}$

(3) for each $x^{*} \in E^{*}$ there exists a set $A\left(x^{*}\right)$ with $\mu_{\Delta}$ measure zero, such that for each $t \notin A\left(x^{*}\right)$,

$$
\left(x^{*} x\right)^{\Delta}(t)=x^{*}\left(f\left(t, x(t), \int_{0}^{t} k(t, s, x(s)) \Delta s\right)\right) .
$$


A continuous function $x: I_{a} \rightarrow E$ is said to be a solution of the problem (3.1) if it satisfies

$$
x(t)=x_{0}+\int_{0}^{t} f\left(z, x(z), \int_{0}^{z} k(z, s, x(s)) \Delta s\right) \Delta z, \quad \text { for every } t \in I_{a} .
$$

Because we consider a new type of integral and a new type of solutions is necessary to prove that each solution $x$ of problem (1.1) is the solution of problem (3.1).

Let $x$ is a continuous solution of (3.1). Fix $x^{*} \in E^{*}$. By definition, $x$ is $\mathrm{ACG}_{*}$ function and $x(0)=x_{0}$. Since, for each $x^{*} \in E^{*}$ and $\mu_{\Delta}$ a.e $t \in I_{a},\left(x^{*} x(t)\right)^{\Delta}=x^{*}\left(x^{\Delta}(t)\right)=$ $x^{*}\left(f\left(t, x(t), \int_{0}^{t} k(t, s, x(s)) \Delta s\right)\right)$ and the last is $\Delta$-HK integrable, so is differentiable $\mu_{\Delta}$ a.e.

Moreover, $\int_{0}^{t} x^{*}\left(f\left(z, x(z), \int_{0}^{z} k(z, s, x(s) \Delta s)\right) \Delta z=\int_{0}^{t}\left(x^{*} x(s)\right)^{\Delta} \Delta s=x^{*}\left(x(t)-x_{0}\right)\right.$. Thus $x(t)$ satisfies (3.1).

Now assume that $y$ is $\mathrm{ACG}_{*}$ function and $y(0)=x_{0}$. By the definition of $\Delta$-HKP integrals there exists an $\mathrm{ACG}_{*}$ function $G$ such that $G(0)=x_{0}$ and

$$
x^{*}\left(G^{\Delta}(t)\right)=x^{*}\left(f\left(t, y(t), \int_{0}^{t} k(t, s, y(s)) \Delta s\right)\right) \mu_{\Delta} \text { a.e. }
$$

Hence

$$
\begin{aligned}
x^{*}(y(t)) & =x_{0}+\int_{0}^{t} x^{*}\left(f\left(z, y(z), \int_{0}^{z} k(z, s, y(s)) \Delta s\right) \Delta z\right) \\
& =x_{0}+\int_{0}^{t} x^{*}\left(G^{\Delta}\right)(s) \Delta s=x_{0}+x^{*}(G(t))-x^{*}(G(0))=x^{*}(G(t)) .
\end{aligned}
$$

We obtain $y=G, \mu_{\Delta}$-a.e. and then $y^{\Delta}(t)=f\left(t, y(t), \int_{0}^{t} k(t, s, y(s)) \Delta s\right)$.

Let

$$
\begin{gathered}
B=\left\{x \in E:\|x\| \leq\left\|x_{0}\right\|+p, p>0\right\}, \\
\widetilde{B}=\left\{x \in\left(C\left(I_{a}, E\right), \omega\right): x(0)=x_{0},\|x\| \leq\left\|x_{0}\right\|+p, p>0\right\} .
\end{gathered}
$$

Moreover, let $F(x)(t)=x_{0}+\int_{0}^{t} f\left(z, x(z), \int_{0}^{z} k(z, s, x(s)) \Delta s\right) \Delta z, t \in I_{a}, K=\{F(x): x \in \widetilde{B}\}$, $K_{1}=\left\{\int_{0}^{z} k(z, s, x(s)) \Delta s: z \in I_{t}=[0, t] \cap T, t \in I_{a}, x \in \widetilde{B}\right\}$.

Theorem 3.3. Assume, that for each uniformly ACG function $x: I_{a} \rightarrow E$ the functions: $k(\cdot, s, x(s)), f\left(\cdot, x(\cdot), \int_{0}^{(\cdot)} k(\cdot, s, x(s)) \Delta s\right)$ are $\Delta$-HKP integrable, $f$ and $k$ are weakly-weakly sequentially continuous functions. Suppose that there exist constants $d_{1}, d_{2}, d_{3}>0$ such that

$$
\beta(f(I \times A \times C)) \leq d_{1} \cdot \beta(A)+d_{2} \cdot \beta(C),
$$

for each subset $A, C$ of $B, I \subset I_{a}$,

$$
\beta(k(I \times I \times X)) \leq d_{3} \cdot \beta(X)
$$


for each subset $X$ of $B, I \subset I_{a}$, where $f(I \times A \times C)=\left\{f\left(t, x_{1}, x_{2}\right):\left(t, x_{1}, x_{2}\right) \in I \times A \times C\right\}, k(I \times$ $I \times X)=\{k(t, s, x):(t, s, x) \in I \times I \times X\}$.

Moreover, let $K$ and $K_{1}$ be equicontinuous, equibounded and uniformly $A C G_{*}$ on $I_{a}$. Then there exists a pseudosolution of the problem (1.1) on $I_{c}$, for some $c \in T, 0<c \leq a$ and $0<c \cdot d_{1}+c^{2}$. $d_{2} \cdot d_{3}<1$.

Proof. Fix an arbitrary $p \geq 0$. Recall, that a set $K$ of continuous functions $F(x) \in K$ defined on a time scale interval $I_{a}$ is equicontinuous on $I_{a}$ if for each $\varepsilon>0$ there exists $\delta>0$ such that $\|F(x)(t)-F(x)(\tau)\|<\varepsilon$ for all $x \in \widetilde{B}$ whenever $|t-\tau|<\delta, t, \tau \in I_{a}$, for each $F(x) \in K$. Thus, for each $\varepsilon>0$ there exists $\delta>0$ such that $\left\|\int_{\tau}^{t} f\left(z, x(z), \int_{0}^{z} k(z, s, x(s)) \Delta s\right) \Delta z\right\|<\varepsilon$ for all $x \in \widetilde{B}$ whenever $|t-\tau|<\delta$ and $t, \tau \in I_{a}$. As a result, there exists a number $c, 0<c \leq a$ such that

$$
\left\|\int_{0}^{t} f\left(z, x(z), \int_{0}^{z} k(z, s, x(s)) \Delta s\right) \Delta z\right\| \leq p, \quad t \in I_{c}, x \in \widetilde{B} .
$$

We will show that the operator $F$ is well defined and maps $\widetilde{B}$ into $\widetilde{B}$.

To see this note for any $x^{*} \in E^{*}$, such that $\left\|x^{*}\right\| \leq 1$, for any $x \in \widetilde{B}$ and $t \in I_{c}$ we have

$$
\begin{aligned}
\left|x^{*} F(x)(t)\right| & \leq\left|x^{*} x_{0}\right|+\left|x^{*} \int_{0}^{t} f\left(z, x(z), \int_{0}^{z} k(z, s, x(s)) d s\right) \Delta z\right| \\
& \leq\left\|x^{*}\right\|\left\|x_{0}\right\|+\left\|x^{*}\right\|\left\|\int_{0}^{t} f\left(z, x(z), \int_{0}^{z} k(z, s, x(s)) d s\right) \Delta z\right\| \leq\left\|x_{0}\right\|+p,
\end{aligned}
$$

so

$$
\sup \left\{\left|x^{*} F(x)(t)\right|: x^{*} \in E^{*},\left\|x^{*}\right\| \leq 1\right\} \leq\left\|x_{0}\right\|+p
$$

and as a result

$$
\|F(x)(t)\| \leq\left\|x_{0}\right\|+p .
$$

Thus $F(x)(t) \in \widetilde{B}$.

We will show, that the operator $F$ is weakly-weakly sequentially continuous. By Lemma 9 of [34] a sequence $x_{n}(\cdot)$ is weakly convergent in $C\left(I_{c}, E\right)$ to $x(\cdot)$ if and only if $x_{n}(t)$ tends weakly to $x(t)$ for each $t \in I_{c}$, so if $x_{n} \stackrel{\omega}{\rightarrow} x$ in $C\left(I_{c}, E\right)$ then $k\left(t, s, x_{n}(s)\right) \stackrel{\omega}{\rightarrow}$ $k(t, s, x(s))$ in $E$ for $t \in I_{c}$ and by Theorem 2.14 (see our assumptions on $K_{1}$ ) we have $\lim _{n \rightarrow \infty} \int_{0}^{t} k\left(z, s, x_{n}(s)\right) d s=\int_{0}^{t} k(z, s, x(s)) d s$ weakly in $E$, for each $t \in I_{c}$. Moreover, because $f$ is weakly-weakly sequentially continuous, we have

$$
f\left(t, x_{n}(t), \int_{0}^{t} k\left(t, s, x_{n}(s)\right) \Delta s\right) \stackrel{\omega}{\longrightarrow} f\left(t, x(t), \int_{0}^{t} k(t, s, x(s)) \Delta s\right)
$$


in $E$, for each $t \in I_{c}$. Thus Theorem 2.14 (see our assumptions on $K$ ) implies $F\left(x_{n}\right)(t) \rightarrow$ $F(x)(t)$ weakly in $E$ for each $t \in I_{c}$ so Lemma 9 of [34] guarantees that $F\left(x_{n}\right) \rightarrow F(x)$ in $C\left(I_{c}, E\right)$ with its weak topology.

Suppose that $V \subset \widetilde{B}$ satisfies the condition $\bar{V}=\overline{\operatorname{conv}}(\{x\} \cup F(V))$. We will prove that $V$ is relatively weakly compact and so (2.1) is satisfied. Since $V \subset \widetilde{B}, F(V) \subset K$. Then $V \subset \bar{V}=$ $\overline{\operatorname{conv}}(\{x\} \cup F(V))$ is equicontinuous. By Lemma 2.17, $t \mapsto v(t)=\beta(V(t))$ is continuous on $I_{c}$.

For fixed $t \in I_{c}$ we divide the interval $[0, t]$ into $m$ parts in the following way $t_{0}=0$,

$$
\begin{gathered}
t_{1}=\sup _{s \in I_{a}}\left\{s: s \geq t_{0}, s-t_{0}<\delta\right\}, \\
t_{2}=\sup _{s \in I_{a}}\left\{s: s \geq t_{1}, s-t_{1}<\delta\right\}, \\
\vdots \\
t_{n}=\sup _{s \in I_{a}}\left\{s: s \geq t_{n-1}, s-t_{n-1}<\delta\right\} .
\end{gathered}
$$

Since $T$ is closed, we have $t_{i} \in I_{a}$. If some $t_{i+1}=t_{i}$ then $t_{i+2}=\inf \left\{t \in T: t \geq t_{i+1}\right\}$.

For fixed $z \in[0, t]$ we divide the interval $[0, z]$ into $m$ parts: $z_{0}=0$,

$$
\begin{gathered}
z_{1}=\sup _{s \in[0, t]}\left\{s: s \geq z_{0}, s-z_{0}<\delta\right\}, \\
z_{2}=\sup _{s \in[0, t]}\left\{s: s \geq z_{1}, s-z_{1}<\delta\right\}, \\
\vdots \\
z_{n}=\sup _{s \in[0, t]}\left\{s: s \geq z_{n-1}, s-z_{n-1}<\delta\right\} \quad \text { such that } \mu_{\Delta}\left(I_{j}\right)=\frac{j z}{m},
\end{gathered}
$$

$j=0,1, \ldots, m, I_{j}=\left[z_{j}, z_{j+1}\right]$.

Let $V\left(\left[z_{j}, z_{j+1}\right]\right)=\left\{u(s): u \in V, z_{j} \leq s \leq z_{j+1}, j=0,1, \ldots, m-1\right\}$. By Lemma 2.17 and the continuity of $v$ there exists $s_{j} \in I_{j}=\left[z_{j}, z_{j+1}\right]$, such that

$$
\beta\left(V\left(\left[z_{j}, z_{j+1}\right]\right)\right)=\sup \left\{\beta(V(s)): z_{j} \leq s \leq z_{j+1}\right\}:=v\left(s_{j}\right) .
$$

By Theorem 2.13 and the properties of the $\Delta$-HKP integral we have for $x \in V$ that

$$
\begin{aligned}
F(x)(t) & =x_{0}+\sum_{i=0}^{m-1} \int_{J_{i}} f\left(z, x(z), \sum_{j=0}^{m-1} \int_{I_{j}} k(z, s, x(s)) \Delta s\right) \Delta z \\
& \in x_{0}+\sum_{i=0}^{m-1} \mu_{\Delta}\left(J_{i}\right) \overline{\operatorname{conv}} f\left(J_{i}, V\left(J_{i}\right), \sum_{j=0}^{m-1}\left(z_{j+1}-z_{j}\right) \overline{\operatorname{conv}} k\left(I_{j}, I_{j}, V\left(I_{j}\right)\right)\right),
\end{aligned}
$$

where $J_{i}=\left[t_{i}, t_{i+1}\right], i=0,1, \ldots, m-1$. 
Using (3.8), (3.9) and properties of the measure of noncompactness we obtain

$$
\begin{aligned}
\beta(F(V)(t)) & \leq \sum_{i=0}^{m-1} \mu_{\Delta}\left(J_{i}\right) \beta\left(f\left(J_{i}, V\left(J_{i}\right), \sum_{j=0}^{m-1} \mu_{\Delta}\left(I_{j}\right) \overline{\operatorname{conv}} k\left(I_{j}, I_{j}, V\left(I_{j}\right)\right)\right)\right) \\
& \leq \sum_{i=0}^{m-1} \mu_{\Delta}\left(J_{i}\right) \beta\left(f\left(J_{i}, V\left(J_{i}\right), \sum_{j=0}^{m-1} \mu_{\Delta}\left(I_{j}\right) \overline{\operatorname{conv}} k\left(I_{j}, I_{j}, V\left(I_{j}\right)\right)\right)\right) \\
& \leq \sum_{i=1}^{m-1} \mu_{\Delta}\left(J_{i}\right) \cdot d_{1} \cdot \beta\left(V\left(J_{i}\right)\right)+\sum_{i=1}^{m-1} \mu_{\Delta}\left(J_{i}\right) \cdot d_{2} \cdot \beta\left(\sum_{j=0}^{m-1} \mu_{\Delta}\left(I_{j}\right) \cdot \overline{\operatorname{conv}} k\left(I_{j}, I_{j}, V\left(I_{j}\right)\right)\right) \\
& \leq \sum_{i=1}^{m-1} \mu_{\Delta}\left(J_{i}\right) \cdot d_{1} \cdot \beta\left(V\left(I_{c}\right)\right)+\sum_{i=1}^{m-1} \mu_{\Delta}\left(J_{i}\right) \cdot d_{2} \cdot \sum_{j=0}^{m-1} \mu_{\Delta}\left(I_{j}\right) \cdot \beta\left(k\left(I_{j}, I_{j}, V\left(I_{j}\right)\right)\right) \\
& \leq \beta\left(V\left(I_{c}\right)\right) \cdot d_{1} \cdot c+\sum_{i=1}^{m-1} \mu_{\Delta}\left(J_{i}\right) \cdot d_{2} \cdot \sum_{j=0}^{m-1} \mu_{\Delta}\left(I_{j}\right) \cdot d_{3} \cdot \beta\left(V\left(I_{j}\right)\right) \\
& \leq \beta\left(V\left(I_{c}\right)\right) \cdot d_{1} \cdot c+\beta\left(V\left(I_{c}\right)\right) \cdot d_{2} \cdot d_{3} \cdot c^{2}=\beta\left(V\left(I_{c}\right)\right)\left(d_{1} \cdot c+d_{2} \cdot d_{3} \cdot c^{2}\right) .
\end{aligned}
$$

Since $\bar{V}=\overline{\operatorname{conv}}(\{x\} \cup F(V)), \beta(V(t))=\beta(\overline{\operatorname{conv}}(\{x\} \cup F(V(t))))$ so $\beta(V(t)) \leq \beta\left(V\left(I_{c}\right)\right)\left(d_{1} \cdot c+\right.$ $\left.d_{2} \cdot d_{3} \cdot c^{2}\right)$, for $t \in I_{c}$.

Using Lemma 2.17 we obtain

$$
\beta\left(V\left(I_{c}\right)\right) \leq \beta\left(V\left(I_{c}\right)\right)\left(d_{1} \cdot c+d_{2} \cdot d_{3} \cdot c^{2}\right) .
$$

Since $0<c \cdot d_{1}+c^{2} \cdot d_{2} \cdot d_{3}<1$ we obtain $v(t)=\beta(V(t))=0$ for $t \in I_{c}$.

Using Ascoli's theorem we have that $V$ is relatively weakly compact.

By Theorem 2.3 the operator $F$ has a fixed point. This means that there exists a pseudosolution of the problem (1.1).

Theorem 3.4. Assume, that for each uniformly ACG* function $x: I_{a} \rightarrow E$, the functions $k(\cdot, s, x(s)), f\left(\cdot, x(\cdot), \int_{0}^{(\cdot)} k(\cdot, s, x(s)) d s\right)$ are $\Delta$-HKP integrable and $k, f$ are weakly-weakly sequentially continuous. Suppose that there exists a constant $d>0$ and a continuous function $c_{1}: I_{a} \rightarrow R_{+}$ such that

$$
\begin{gathered}
\beta(f(I, A, C)) \leq d \cdot \beta(C), \quad \text { for each } A, C \subset B, I \subset I_{a}, \\
\beta(k(I, I, X)) \leq \sup _{s \in I} c_{1}(s) \beta(X), \quad \text { for each } X \subset B, I \subset I_{a},
\end{gathered}
$$


where

$$
\begin{gathered}
f(I, A, C)=\left\{f\left(t, x_{1}, x_{2}\right):\left(t, x_{1}, x_{2}\right) \in I \times A \times C\right\}, \\
k(I, I, X)=\{k(t, s, x):(t, s, x) \in I \times I \times X\} .
\end{gathered}
$$

Moreover, let $K$ and $K_{1}$ be equicontinuous and uniformly $A C G^{*}$ on $I_{a}$. Then there exists a pseudo solution of the problem (1.1) on $I_{c}$, for some $c \in T, 0<c \leq a$.

Proof. The first part of the proof is the same as that of the proof of the previous theorem. It remains to show the relative compactness of $V$, where $V$ is defined in Theorem 3.3. In this case notice for $t \in I_{c}$ and $z_{j}$ as in Theorem 3.3 we have

$$
\begin{aligned}
\beta(V(t)) & \leq \sum_{i=0}^{m-1} \mu_{\Delta}\left(J_{i}\right) \cdot d \cdot \beta\left(\sum_{j=0}^{m-1} \mu_{\Delta}\left(I_{j}\right) \cdot \overline{\operatorname{conv}} k\left(I_{j}, I_{j}, V\left(I_{j}\right)\right)\right) \\
& \leq \sum_{i=0}^{m-1} \mu_{\Delta}\left(J_{i}\right) \cdot d \cdot \sum_{j=0}^{m-1} \mu_{\Delta}\left(I_{j}\right) \cdot \beta\left(k\left(I_{j}, I_{j}, V\left(I_{j}\right)\right)\right) \\
& \leq \sum_{i=0}^{m-1} \mu_{\Delta}\left(J_{i}\right) \cdot d \cdot \sum_{j=0}^{m-1} \mu_{\Delta}\left(I_{j}\right) \cdot \sup _{s \in I_{j}} c_{1}(s) \beta\left(V\left(I_{j}\right)\right) .
\end{aligned}
$$

Let $\sup _{s \in I_{j}} c_{1}(s)=c_{1}\left(p_{j}\right), p_{j} \in I_{j}$. Then

$$
\begin{aligned}
\beta(V(t)) & \leq c \cdot d \cdot \sum_{j=0}^{m-1} \mu_{\Delta}\left(I_{j}\right) \cdot c_{1}\left(p_{j}\right) v\left(s_{j}\right) \\
& =c \cdot d\left(\sum_{j=0}^{m-1} \mu_{\Delta}\left(I_{j}\right) \cdot c_{1}\left(p_{j}\right) v\left(p_{j}\right)+\sum_{j=0}^{m-1} \mu_{\Delta}\left(I_{j}\right)\left(c_{1}\left(p_{j}\right)\left(v\left(s_{j}\right)-v\left(p_{j}\right)\right)\right)\right) .
\end{aligned}
$$

Fix $\varepsilon>0$. From the equicontinuity of $V$ we may choose $m$ large enough so that $v\left(s_{j}\right)-v\left(p_{j}\right)<\varepsilon$ and so

$$
\begin{aligned}
\beta(V(t)) & \leq c \cdot d\left(\sum_{j=0}^{m-1} \mu_{\Delta}\left(I_{j}\right) \cdot c_{1}\left(p_{j}\right) v\left(p_{j}\right)+\sum_{j=0}^{m-1} \frac{z}{m} c_{1}\left(p_{j}\right) \cdot \varepsilon\right) \\
& \leq c \cdot d\left(\sum_{j-0}^{m-1} \mu_{\Delta}\left(I_{j}\right) c_{1}\left(p_{j}\right) v\left(p_{j}\right)+z \cdot \varepsilon \cdot \max _{0 \leq k \leq m-1} c_{1}\left(p_{k}\right)\right) .
\end{aligned}
$$

Since $\varepsilon>0$ is arbitrary small and $z \cdot \max _{0 \leq k \leq m-1} c_{1}\left(p_{k}\right)$ is bounded, we have that $z \cdot \varepsilon$. $\max _{0 \leq k \leq m-1} c_{1}\left(p_{k}\right)$ is arbitrary small. Therefore

$$
v(t)=\beta(V(t)) \leq c \cdot d \cdot \int_{0}^{t} c_{1}(s) v(s) \Delta s, \quad t \in I_{c} .
$$


Thus, by Gronwall's inequality for $\Delta$-integrals [5] we have that

$$
v(t)=\beta(V(t))=0, \quad \text { for } t \in I_{c} .
$$

Using Ascoli's theorem we deduce that $V$ is relatively weakly compact.

By Theorem 2.14 the operator $F$ has a fixed point. This means that there exists a pseudo solution of the problem (1.1).

Remark 3.5. The conditions in Theorems 3.3 and 3.4 can be also generalized to the Sadovskii condition [28], the Szufla condition [49], and the others and $\beta$ can be replaced by some axiomatic measure of weak noncompactness.

\section{References}

[1] V. Kac and P. Cheung, Quantum Calculus, Springer, New York, NY USA, 2002.

[2] S. Hilger, Ein Maßkettenkalkül mit Anvendung auf Zentrumsmannigfaltigkeiten, Ph.D. thesis, Universität at Würzburg, Würzburg, Germany, 1988.

[3] R. P. Agarwal and M. Bohner, "Basic calculus on time scales and some of its applications," Results in Mathematics, vol. 35, no. 1-2, pp. 3-22, 1999.

[4] R. Agarwal, M. Bohner, and A. Peterson, "Inequalities on time scales: a survey," Mathematical Inequalities \& Applications, vol. 4, no. 4, pp. 535-557, 2001.

[5] E. Akin-Bohner, M. Bohner, and F. Akin, "Pachpate inequalities on time scale," Journal of Inequalities in Pure and Applied Mathematics, vol. 6, no. 1, article no. 6, 2005.

[6] B. Aulbach and S. Hilger, "Linear dynamic processes with inhomogeneous time scale," in Nonlinear Dynamics and Quantum Dynamical Systems, vol. 59, pp. 9-20, Akademie, Berlin, Germany, 1990.

[7] M. Bohner and A. Peterson, Dynamic Equations on Time Scales, Birkhäuser, Boston, Mass, USa, 2001.

[8] M. Bohner and A. Peterson, Advances in Dynamic Equations on Time scales, Birkhäuser, Boston, Mass, USA, 2003.

[9] G. S. Guseinov, "Integration on time scales," Journal of Mathematical Analysis and Applications, vol. 285, no. 1, pp. 107-127, 2003.

[10] S. Hilger, "Analysis on measure chains-a unified approach to continuous and discrete calculus," Results in Mathematics, vol. 18, no. 1-2, pp. 18-56, 1990.

[11] B. Kaymakcalan, V. Lakshmikantham, and S. Sivasundaram, Dynamic Systems on Measure Chains, vol. 370 of Mathematics and Its Applications, Kluwer Academic Publishers, Dordrecht, The Netherlands, 1996.

[12] L. Erbe and A. Peterson, "Green's functions and comparison theorems for differential equations on measure chains," Dynamics of Continuous, Discrete and Impulsive Systems, vol. 6, no. 1, pp. 121-137, 1999.

[13] D. Franco, "Green's functions and comparison results for impulsive integrodifferential equations," Nonlinear Analysis: Theory, Methods \& Applications, vol. 47, pp. 5723-5728, 2001.

[14] D. Guo, "Initial value problems for second-order integro-differential equations in Banach spaces," Nonlinear Analysis. Theory, Methods E Applications, vol. 37, no. 3, pp. 289-300, 1999.

[15] D. Guo and X. Liu, "Extremal solutions of nonlinear impulsive integrodifferential equations in Banach spaces," Journal of Mathematical Analysis and Applications, vol. 177, no. 2, pp. 538-552, 1993.

[16] L. S. Liu, C. Wu, and F. Guo, "A unique solution of initial value problems for first order impulsive integro-differential equations of mixed type in Banach spaces," Journal of Mathematical Analysis and Applications, vol. 275, no. 1, pp. 369-385, 2002.

[17] X. Liu and D. Guo, "Initial value problems for first order impulsive integro-differential equations in Banach spaces," Communications on Applied Nonlinear Analysis, vol. 2, no. 1, pp. 65-83, 1995.

[18] H. Lu, "Extremal solutions of nonlinear first order impulsive integro-differential equations in Banach spaces," Indian Journal of Pure and Applied Mathematics, vol. 30, no. 11, pp. 1181-1197, 1999.

[19] G.-X. Song, "Initial value problems for systems of integrodifferential equations in Banach spaces," Journal of Mathematical Analysis and Applications, vol. 264, no. 1, pp. 68-75, 2001. 
[20] H.-K. Xu and J. J. Nieto, "Extremal solutions of a class of nonlinear integro-differential equations in Banach spaces," Proceedings of the American Mathematical Society, vol. 125, no. 9, pp. 2605-2614, 1997.

[21] S. N. Zhang, "The unique existence of periodic solutions of linear Volterra difference equations," Journal of Mathematical Analysis and Applications, vol. 193, no. 2, pp. 419-430, 1995.

[22] C. Corduneanu, Integral Equations and Applications, Cambridge University Press, Cambridge, UK, 1991.

[23] Y. Xing, M. Han, and G. Zheng, "Initial value problem for first-order integro-differential equation of Volterra type on time scales," Nonlinear Analysis. Theory, Methods \& Applications, vol. 60, no. 3, pp. 429-442, 2005.

[24] V. B. Kolmanovskii, E. Castellanos-Velasco, and J. A. Torres-Muñoz, "A survey: stability and boundedness of Volterra difference equations," Nonlinear Analysis. Theory, Methods E Applications, vol. 53, no. 7-8, pp. 861-928, 2003.

[25] Y. Xing, W. Ding, and M. Han, "Periodic boundary value problems of integro-differential equation of Volterra type on time scales," Nonlinear Analysis. Theory, Methods E Applications, vol. 68, no. 1, pp. 127-138, 2008.

[26] A. Peterson and B. Thompson, "Henstock-Kurzweil delta and nabla integrals," Journal of Mathematical Analysis and Applications, vol. 323, no. 1, pp. 162-178, 2006.

[27] S. Avsec, B. Bannish, B. Johnson, and S. Meckler, "The Henstock-Kurzweil delta integral on unbounded time scales," Panamerican Mathematical Journal, vol. 16, no. 3, pp. 77-98, 2006.

[28] B. J. Pettis, "On integration in vector spaces," Transactions of the American Mathematical Society, vol. 44, no. 2, pp. 277-304, 1938.

[29] M. Cichon, "On integrals of vector-valued functions on time scales," to appear.

[30] A. Szép, "Existence theorem for weak solutions of ordinary differential equations in reflexive Banach spaces," Studia Scientiarum Mathematicarum Hungarica, vol. 6, pp. 197-203, 1971.

[31] E. Cramer, V. Lakshmikantham, and A. R. Mitchell, “On the existence of weak solutions of differential equations in nonreflexive Banach spaces," Nonlinear Analysis. Theory, Methods E Applications, vol. 2, no. 2, pp. 169-177, 1978.

[32] I. Kubiaczyk, "Kneser type theorems for ordinary differential equations in Banach spaces," Journal of Differential Equations, vol. 45, no. 2, pp. 139-146, 1982.

[33] I. Kubiaczyk and S. Szufla, "Kneser's theorem for weak solutions of ordinary differential equations in Banach spaces," Publications de l'Institut Mathématique, vol. 32, pp. 99-103, 1982.

[34] A. R. Mitchell and C. Smith, "An existence theorem for weak solutions of differential equations in Banach spaces," in Nonlinear Equations in Abstract Spaces, V. Lakshmikantham, Ed., pp. 387-404, Academic Press, New York, NY, USA, 1978.

[35] S. Szufla, "Kneser's theorem for weak solutions of ordinary differential equations in reflexive Banach spaces," Bulletin of the Polish Academy of Sciences. Mathematics, vol. 26, no. 5, pp. 407-413, 1978.

[36] M. Cichoń and I. Kubiaczyk, "On the set of solutions of the Cauchy problem in Banach spaces," Archiv der Mathematik, vol. 63, no. 3, pp. 251-257, 1994.

[37] T. Kulik and C. C. Tisdell, "Volterra integral equations on time scales: basic qualitative and quantitative results with applications to initial value problems on unbounded domains," International Journal of Difference Equations, vol. 3, no. 1, pp. 103-133, 2008.

[38] Ravi P. Agarwal and D. O'Regan, "Difference equations in abstract spaces," Australian Mathematical Society Journal. Series A, vol. 64, no. 2, pp. 277-284, 1998.

[39] M. Dawidowski, I. Kubiaczyk, and J. Morchało, "A discrete boundary value problem in Banach spaces," Glasnik Matematički, vol. 36, no. 2, pp. 233-239, 2001.

[40] I. Kubiaczyk, J. Morchało, and A. Puk, "A discrete boundary value problem with parameters in Banach space," Glasnik Matematički, vol. 38, no. 2, pp. 299-309, 2003.

[41] J. M. Ball, "Weak continuity properties of mappings and semigroups," Proceedings of the Royal Society of Edinburgh. Section A, vol. 72, no. 4, pp. 275-280, 1975.

[42] I. Kubiaczyk, "On a fixed point theorem for weakly sequentially continuous mappings," Discussiones Mathematicae. Differential Inclusions, vol. 15, no. 1, pp. 15-20, 1995.

[43] A. Cabada and D. R. Vivero, "Expression of the Lebesgue $\Delta$-integral on time scales as a usual Lebesgue integral: application to the calculus of $\Delta$-antiderivatives," Mathematical and Computer Modelling, vol. 43, no. 1-2, pp. 194-207, 2006.

[44] R. Henstock, The General Theory of Integration, Oxford Mathematical Monographs, The Clarendon Press, Oxford, UK, 1991.

[45] A. Sikorska-Nowak, "Convergence theorem for the delta Henstock-Kurzweil-Pettis integrals," to appear. 
[46] J. Banaś and K. Goebel, Measures of Noncompactness in Banach Spaces, vol. 60, Marcel Dekker, New York, Ny, USA, 1980.

[47] I. Kubiaczyk, "On the existence of solutions of differential equations in Banach spaces," Bulletin of the Polish Academy of Sciences. Mathematics, vol. 33, no. 11-12, pp. 607-614, 1985.

[48] A. Ambrosetti, "Un teorema di esistenza per le equazioni differenziali negli spazi di Banach," Rendiconti del Seminario Matematico della Università di Padova, vol. 39, pp. 349-361, 1967.

[49] S. Szufla, "Measure of non-compactness and ordinary differential equations in Banach spaces," Bulletin of the Polish Academy of Sciences. Mathematics, vol. 19, pp. 831-835, 1971. 


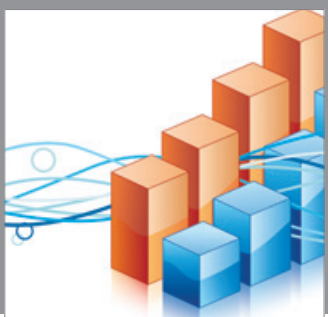

Advances in

Operations Research

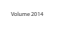

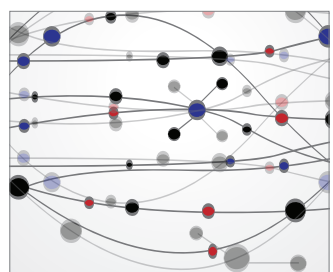

\section{The Scientific} World Journal
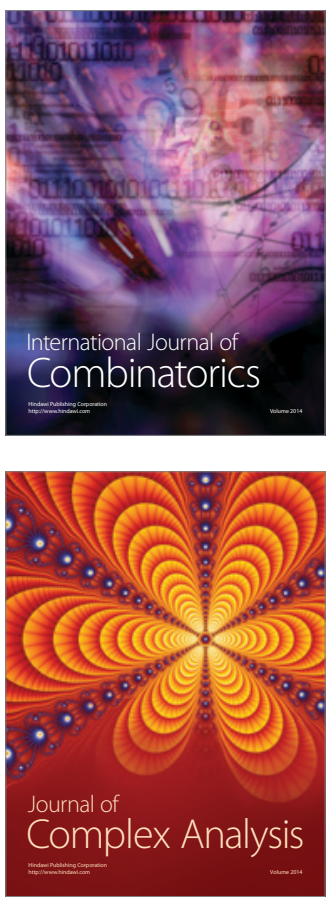

International Journal of

Mathematics and

Mathematical

Sciences
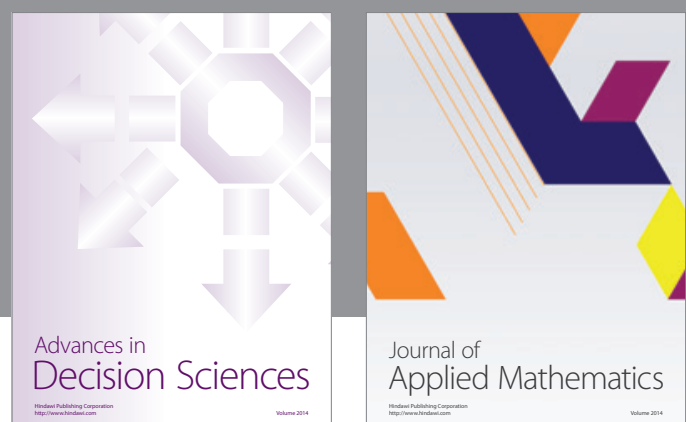

Journal of

Applied Mathematics
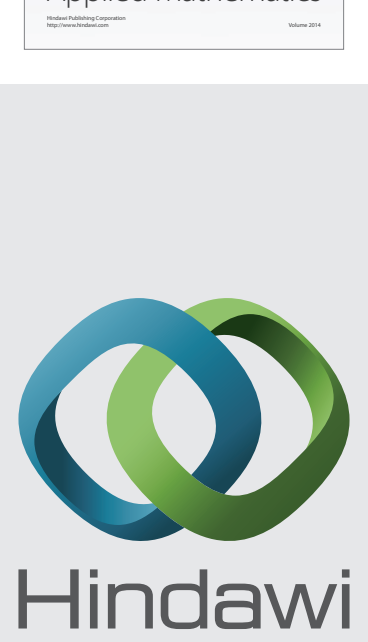

Submit your manuscripts at http://www.hindawi.com
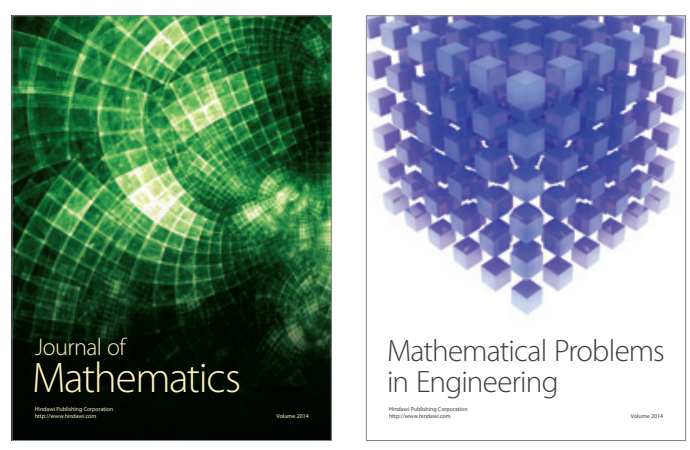

Mathematical Problems in Engineering
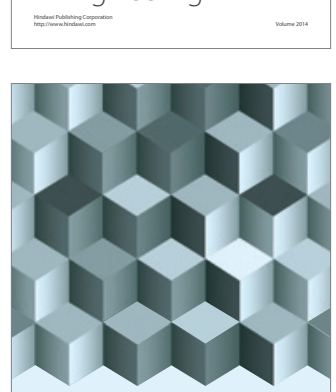

Journal of

Function Spaces
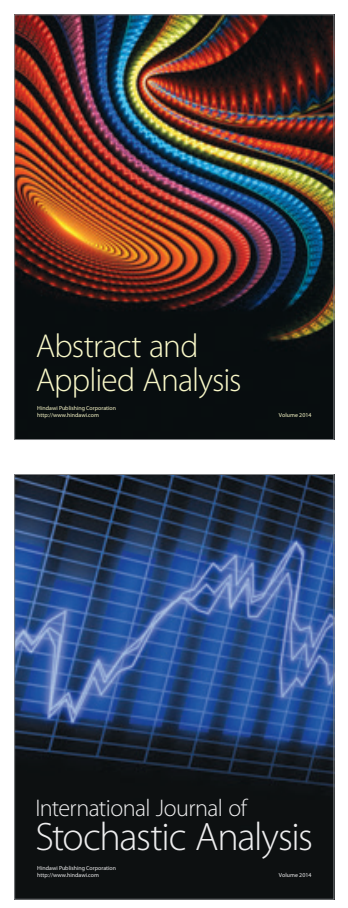

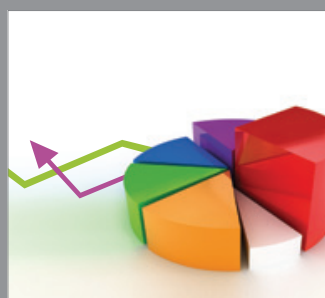

ournal of

Probability and Statistics

Promensencen
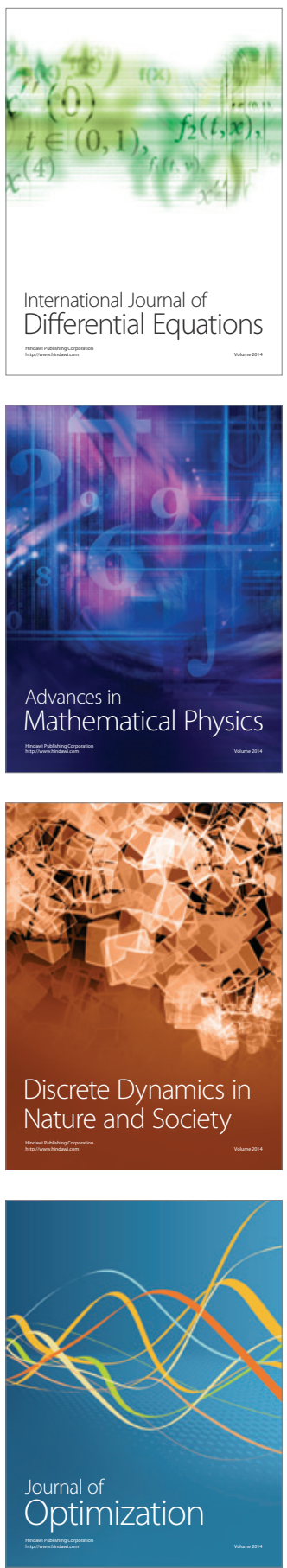